\title{
BMJ Open Ambulatory oxygen for treatment of exertional hypoxaemia in pulmonary fibrosis (PFOX trial): a randomised controlled trial
}

\author{
Anne E Holland (D) , ,2,3,4 Tamera Corte, ${ }^{4,5,6}$ Daniel C Chambers, ${ }^{4,7,8}$ \\ Andrew J Palmer, ${ }^{4,9,10}$ Magnus Per Ekström, ${ }^{11}$ lan Glaspole, ${ }^{2,4,12}$ \\ Nicole S L Goh, ${ }^{3,13,14}$ Graham Hepworth, ${ }^{15}$ Yet H Khor, ${ }^{3,12,13,14}$ Mariana Hoffman, ${ }^{2}$ \\ Ross Vlahos, ${ }^{16}$ Magnus Sköld, ${ }^{17,18}$ Leona Dowman, ${ }^{2,3,14,19}$ Lauren K Troy, ${ }^{5,6}$ \\ Jyotika D Prasad, ${ }^{12,20}$ James Walsh, ${ }^{21}$ Christine F McDonald ${ }^{3,13,14}$
}

To cite: Holland AE, Corte T, Chambers DC, et al. Ambulatory oxygen for treatment of exertional hypoxaemia in pulmonary fibrosis (PFOX trial): a randomised controlled trial. BMJ Open 2020;10:e040798. doi:10.1136/ bmjopen-2020-040798

- Prepublication history and additional material for this paper are available online. To view these files, please visit the journal online (http://dx.doi. org/10.1136/bmjopen-2020040798).

Received 22 May 2020 Revised 22 0ctober 2020 Accepted 15 November 2020

Check for updates

(C) Author(s) (or their employer(s)) 2020. Re-use permitted under CC BY-NC. No commercial re-use. See rights and permissions. Published by BMJ.

For numbered affiliations see end of article.

Correspondence to

Dr Anne E Holland;

a.holland@alfred.org.au

\section{ABSTRACT}

Introduction Interstitial lung diseases are characterised by scarring of lung tissue that leads to reduced transfer of oxygen into the blood, decreased exercise capacity and premature death. Ambulatory oxygen therapy may be used to treat exertional oxyhaemoglobin desaturation, but there is little evidence to support its efficacy and there is wide variation in clinical practice. This study aims to compare the clinical efficacy and cost-effectiveness of ambulatory oxygen versus ambulatory air in people with fibrotic interstitial lung disease and exertional desaturation.

Methods and analysis A randomised, controlled trial with blinding of participants, clinicians and researchers will be conducted at trial sites in Australia and Sweden. Eligible participants will be randomised 1:1 into two groups. Intervention participants will receive ambulatory oxygen therapy using a portable oxygen concentrator (POC) during daily activities and control participants will use an identical POC modified to deliver air. Outcomes will be assessed at baseline, 3 months and 6 months. The primary outcome is change in physical activity measured by number of steps per day using a physical activity monitor (StepWatch). Secondary outcomes are functional capacity (6-minute walk distance), health-related quality of life (St George Respiratory Questionnaire, EQ-5D-5L and King's Brief Interstitial Lung Disease Questionnaire), breathlessness (Dyspnoea-12), fatigue (Fatigue Severity Scale), anxiety and depression (Hospital Anxiety and Depression Scale), physical activity level (GENEActive), oxygen saturation in daily life, POC usage, and plasma markers of skeletal muscle metabolism, systematic inflammation and oxidative stress. A cost-effectiveness evaluation will also be undertaken.

Ethics and dissemination Ethical approval has been granted in Australia by Alfred Hospital Human Research Ethics Committee (HREC/18/Alfred/42) with governance approval at all Australian sites, and in Sweden (Lund Dnr: 2019-02963). The results will be published in peerreviewed scientific journals, presented at conferences and disseminated to consumers in publications for lay audiences.

\section{Strengths and limitations of this study}

- This multisite randomised controlled trial will recruit participants with fibrotic interstitial lung disease (flLD) from six centres in two countries.

- The control group will receive ambulatory oxygen using a sham portable concentrator, identical to the device used by the intervention group except that it will deliver air rather than oxygen, thus allowing effective blinding of participants, health professionals and researchers.

- The primary outcome is physical activity in daily life, an outcome that is meaningful to people living with fILD, and the study includes a comprehensive economic analysis to inform future funding and policy decisions.

- Participants will be followed for 6 months, so longer term outcomes of ambulatory oxygen will not be evaluated.

- It is possible that portable concentrators may not meet the oxygen needs of patients with severe exertional desaturation or rapidly progressive disease, requiring transition to other devices or to long-term oxygen therapy during the trial.

Trial registration number ClinicalTrials.gov Registry (NCT03737409).

\section{BACKGROUND}

The interstitial lung diseases (ILDs) are a group of over 200 debilitating conditions characterised by scarring of lung tissue. Stiffening of the lungs leads to reduced transfer of oxygen into the blood, decreased exercise capacity and premature death. One of the most common types of ILD is idiopathic pulmonary fibrosis (IPF), which confers a particularly poor prognosis, but there are a range of other chronic fibrosing ILDs (fILD) 
that are characterised by similar biological and clinical features. ${ }^{1}$ Exertional desaturation (low oxygen levels on exertion) is a key feature of fILD, predicting poor outcomes including pulmonary hypertension ${ }^{2} 3$ and increased mortality. ${ }^{4}$ Exertional desaturation is also associated with reduced physical activity in daily life. ${ }^{5}$ The relationship of exertional desaturation to poor outcomes provides a rationale for correction of oxygen levels during exercise, to improve both daily functioning and long-term prognosis.

Ambulatory oxygen therapy, defined as the use of supplemental oxygen during exercise and activities of daily living, has historically been used to optimise oxygen saturation and exercise capacity ${ }^{6}$ and is sometimes used to correct exertional desaturation. Access to ambulatory oxygen therapy varies across the world ${ }^{7-9}$ and recommendations regarding ambulatory oxygen therapy in international clinical guidelines are contradictory, ${ }^{610}$ reflecting a lack of robust science to guide policy and practice. We have previously shown that ambulatory oxygen therapy may improve oxygen delivery to skeletal muscle, ${ }^{11}$ providing a potential mechanism by which oxygen therapy could improve exercise capacity and daily physical activity, outcomes that are strongly linked to health-related quality of life (HRQL) and survival. ${ }^{512}$ However, our systematic review found no parallel-group randomised controlled trials of ambulatory oxygen therapy for fILD. ${ }^{13}$ Recently, a crossover trial with a 2-week treatment period demonstrated that ambulatory oxygen increased quality of life in people with fILD. ${ }^{14}$ However, the study was unblinded, and longer term outcomes beyond 2 weeks are unknown.

A key issue affecting trials of oxygen therapy is adherence to treatment. Ambulatory oxygen therapy has traditionally been delivered using portable cylinders, which are heavy and awkward to transport. In a randomised controlled trial of ambulatory oxygen therapy conducted in patients with obstructive lung disease, ${ }^{15}$ the use of cylinder gas averaged just $40 \mathrm{~min}$ per day in both groups. Portable oxygen concentrators (POCs) have emerged as a potential solution to the problems of finite cylinder life and poor transportability. A concentrator is constantly extracting oxygen from air, so oxygen supply continues as long as the battery is charged. During exercise testing, ambulatory oxygen therapy delivered using a POC had similar effects to a standard portable cylinder and was preferred by patients. ${ }^{16}$ The POC provides the opportunity to deliver a robust sham treatment to a control group, as it can be modified to deliver air and thus ensure blinding of participants, health professionals and investigators. However, whether a POC can effectively deliver ambulatory oxygen therapy in daily life for patients with fILD is unknown.

People living with fILD have identified the 'reduction in bureaucratic barriers to oxygen provision' as a major unmet medical need, ${ }^{17}$ highlighting the importance of this treatment to patients. However, the burdens of ambulatory oxygen therapy are also well described. While some people with fILD reported that ambulatory oxygen therapy improved their confidence and feeling of control, this was offset by the embarrassment and stigma associated with oxygen use. $^{18} 19$ Some reported unmet expectations for symptom relief from ambulatory oxygen therapy, although most felt it helped them to be more active. ${ }^{19}$ Oxygen therapy is a key driver of outpatient costs for fILD, ${ }^{20}$ but at present there are no data to confirm whether ambulatory oxygen therapy conveys improvements in patient-centred outcomes that outweigh the costs to patients, the healthcare system and society.

We hypothesise that (1) ambulatory oxygen therapy delivered using a POC will provide clinically significant improvements in physical activity (primary outcome), symptoms and HRQL; and (2) ambulatory oxygen therapy via POC will be cost-effective compared with ambulatory air.

\section{METHODS AND ANALYSIS \\ Design}

A randomised controlled superiority trial, with blinding of participants, clinicians and researchers, in 260 people with fILD who desaturate during walking. The trial will be conducted in Australia and Sweden, with trial sites detailed on the study registration at ClinicalTrials.gov. Participants will be randomised 1:1 into two groups: group 1: ambulatory oxygen therapy using a POC (oxygen group), group 2: sham therapy using an identical POC (air group). The allocated treatment will be delivered for 6 months. We have previously demonstrated the feasibility of the trial methodology in a pilot study. ${ }^{21}$ Figure 1 shows the participant flow through the trial. The overview of the study procedures follows the Standard Protocol Items: Recommendations for Interventional Trials (SPIRIT) checklist ${ }^{22}$ (see online supplemental file 1).

\section{Participants}

People with fILD who receive their care at the study sites will be invited to participate. Patients will be eligible for inclusion if they are (1) aged 18 years and over; (2) have a physician-confirmed diagnosis of fILD, such as IPF, connective tissue disease-associated ILD, fibrotic hypersensitivity pneumonitis, idiopathic non-specific interstitial pneumonia, unclassifiable idiopathic interstitial pneumonia, environmental/occupational lung disease or sarcoidosis, with features of diffuse fILD of $>10 \%$ extent on high-resolution CT, with ILD being the predominant pathological process $^{23}$; (3) have had stable pharmacotherapy over the last 3 months; and (4) exhibit exertional desaturation, defined as oxyhaemoglobin saturation $\left(\mathrm{SpO}_{2}\right) \leq 88 \%$ for at least 10 consecutive seconds during a 6 -minute walk test (6MWT) performed on room air. Only fILD will be included as these conditions are often characterised by chronic progressive fibrosis, as opposed to other ILDs with differing pathophysiology (eg, inflammatory or granulomatous) where different mechanisms may underlie exertional desaturation. 


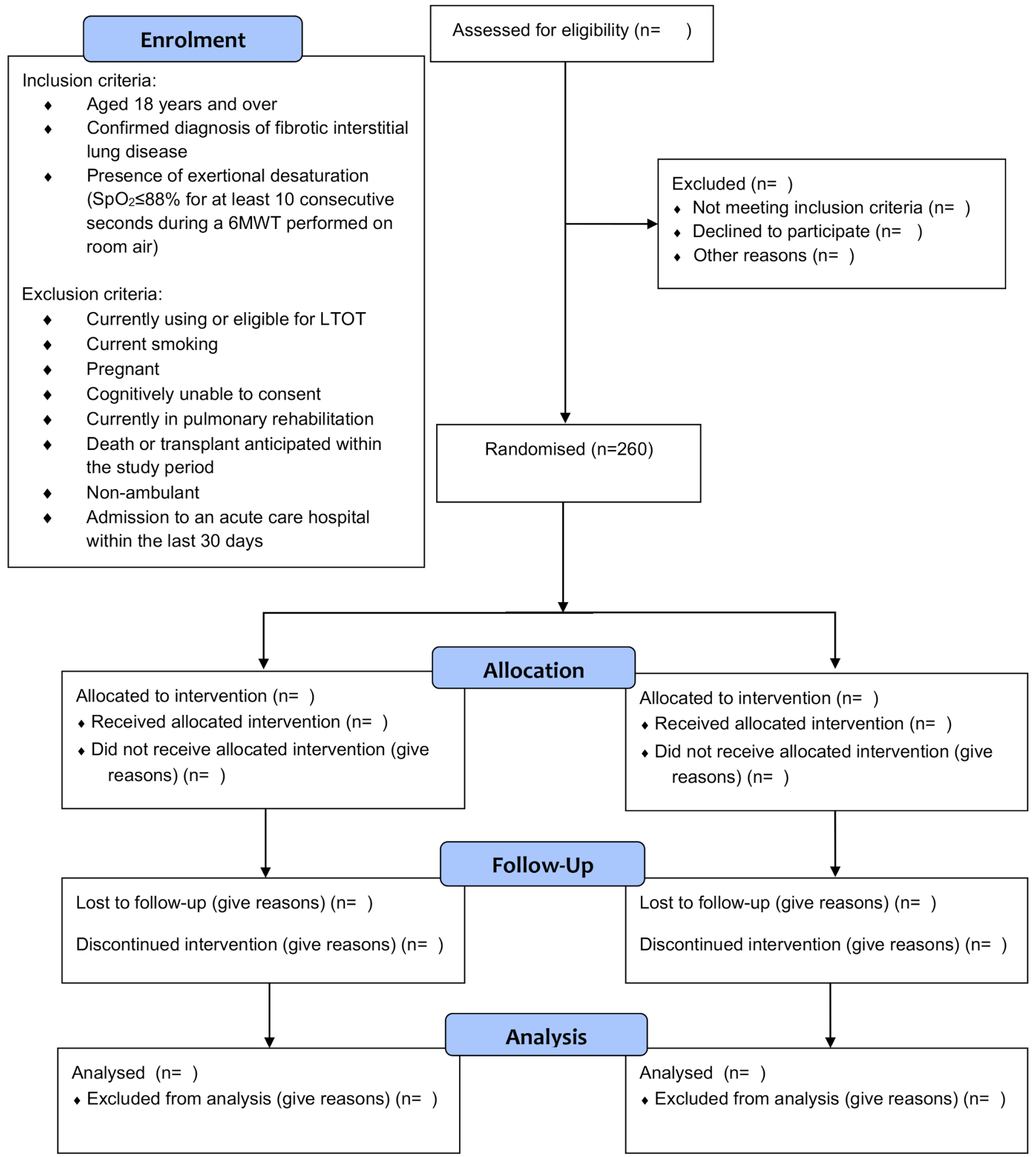

Figure 1 Study flow. 6MWT, 6-minute walk test; LTOT, long-term oxygen therapy; $\mathrm{SpO}_{2}$, oxyhaemoglobin saturation.

Participants will be excluded if they: (1) are currently using or eligible for long-term oxygen therapy (LTOT), with eligibility defined as arterial oxygen pressure $\leq 55 \mathrm{~mm}$ $\mathrm{Hg}$ at rest on room air, or 56-59 $\mathrm{mm} \mathrm{Hg}$ with evidence of right heart failure ${ }^{10}$ as it is not ethical to withhold oxygen therapy in this group for whom it is strongly recommended $^{24}$; (2) are current smokers, due to the risk of oxygen use near flames; (3) have predominantly obstructive lung disease, with forced expiratory ratio less than the lower limit of normal; (4) are pregnant; (5) are cognitively unable to consent; (6) are non-ambulant; (7) have been admitted to an acute care hospital within the last 30 days; or (8) if death or transplant is anticipated within the study period. Participants currently participating in 
pulmonary rehabilitation will not be enrolled, and participation in pulmonary rehabilitation during the 6-month trial period will be avoided where possible, as this may impact on both primary and secondary outcomes.

\section{Recruitment}

Potential participants will be identified by their treating healthcare team. If the participant is interested in obtaining more detailed information, they will be contacted by the trial coordinator or site coordinator, who is not in a dependent relationship with the patient and will provide further information. Patients will be informed that participation in the study is voluntary, their decision about participation will not affect their treatment or relationship with their healthcare team, their data will be held securely and they will not be identified in any study publications. Only patients who provide written, informed consent will undertake the study procedures outlined in this protocol.

\section{Patient and public involvement}

We interviewed patients with fILD and physicians who cared for them, in order to understand experiences and role of oxygen therapy ${ }^{719}$ Patients emphasised the need for oxygen devices that were lighter and easier to use. Our subsequent work showed that patients preferred using a POC over traditional oxygen cylinders. ${ }^{16}$ In our feasibility trial we interviewed participants about their experiences of trial participation. Participants expressed positive experiences of the study and stated they would recommend such trial participation to others with ILD. ${ }^{21}$ These experiences underpinned the design of the protocol for the current trial.

\section{Randomisation}

Randomisation will occur following completion of the baseline assessment, including measures of physical activity. Participants will be randomly allocated to groups using a computer-generated, permuted block randomisation schedule with stratification for (1) desaturation during $6 \mathrm{MWT}(<80 \%$ vs $\geq 80 \%)$ as this is a powerful predictor of physical activity, HRQL and mortality, ${ }^{4} 25$ and (2) site of recruitment. Sequence generation will be performed by an individual independent of the research team and the allocation sequence will be concealed using a secure online randomisation service.

\section{Interventions}

All participants will be informed that the aim of using a POC is to assist them to be more active, with fewer symptoms. They will be encouraged to use the POC at all times when they are moving about, including walking at home or in the community, during exercise or during other activities. The POC should not be used when sitting still or sleeping. Written and verbal education will be provided. Participants will be encouraged to use their allocated POC during physical activity for the 6-month study period. Participants will be provided with a standard POC carry bag, worn over one shoulder, but will have the option to use a backpack if required. The method by which each participant chooses to carry their POC will be recorded. Informed by our pilot study, the Inogen One G3 HF POC will be used at its maximum pulse flow setting of 5 for both groups, as this delivered similar oxygen saturation during walking to a portable cylinder delivering $5 \mathrm{~L} / \mathrm{min}$ of oxygen on continuous flow. ${ }^{16}$ No titration of flow rates will be performed, in order to maintain blinding of clinicians, researchers and participants.

All participants will be contacted monthly by telephone by a blinded investigator to encourage POC use and answer any questions. These calls will also collect adverse events, healthcare utilisation data for economic analyses and information on concurrent therapies, including changes to medications. It is likely that some participants will commence LTOT during the 6-month study period. This will occur if participants meet the usual LTOT eligibility criteria ${ }^{10}$ and it is recommended by their treating physician. On commencement of LTOT the participant will cease using the allocated POC. The number of participants in each group who commence LTOT will be recorded, outcome measures will be collected as per the trial protocol and data will be analysed according to allocated treatment group, as per intention-to-treat principles. Similarly, if a participant deteriorates during the 6-month study period then they may commence new/ additional pharmacotherapies at their physician's discretion. The number of participants in each group who commence new pharmacotherapies and their nature will be recorded, outcome measures will be collected as per the trial protocol and data will be analysed according to allocated treatment group, as per intention-to-treat principles. The hours of usage downloaded from POC flash memory will be evaluated every 3 months. Reason for cessation of therapy will be recorded where relevant (patient request, commencement of LTOT, other).

\section{Blinding}

Participants, clinicians and researchers will be blinded to group allocation. The Inogen One G3 HF POCs for ambulatory oxygen therapy and air groups will be identical in appearance, display, weight and operation, with the only difference being the gas delivered. The POCs will be coded by the distributor, who will not be involved in trial conduct. We successfully used this system to maintain blinding in a previous trial using gas cylinders in chronic obstructive pulmonary disease ${ }^{15}$ and our feasibility trial did not identify any safety issues in randomising participants with fILD and exertional desaturation to POCs delivering air. ${ }^{21}$ The intervention code will only be available to the randomisation centre. All participants will be advised against measuring oxygen saturation at home during the duration of the trial, as this does not represent usual clinical practice in any of the centres and may unblind the participants. At the conclusion of the trial, participants will be asked two questions to evaluate the success of blinding: (1) Which treatment do you think you were receiving, oxygen or air? and (2) Did you have 
Table 1 Assessment schedule

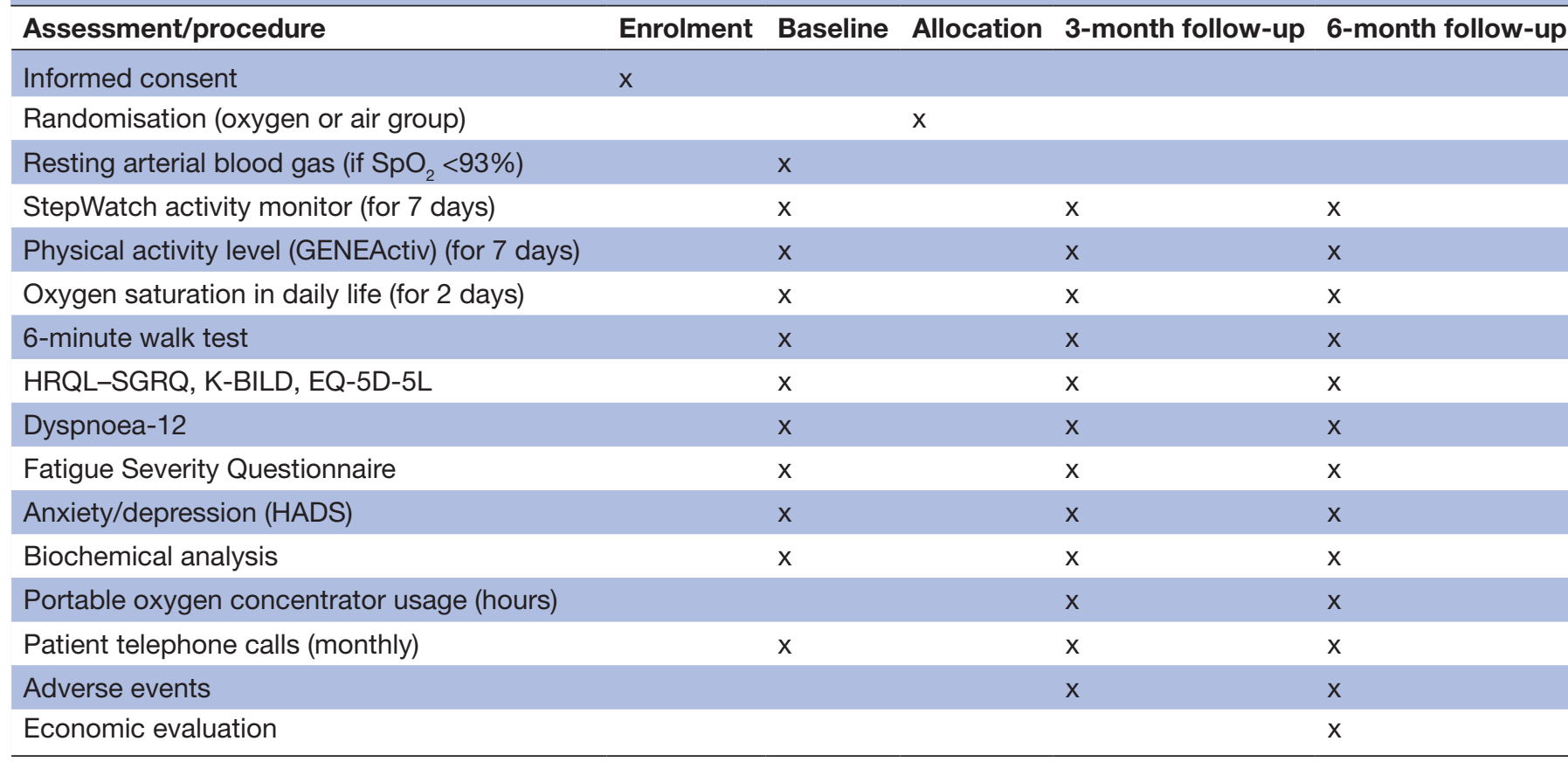

HADS, Hospital Anxiety and Depression Scale; HRQL, health-related quality of life; K-BILD, King's Brief Interstitial Lung Disease questionnaire; SGRQ, St George's Respiratory Questionnaire; $\mathrm{SpO}_{2}$, oxyhaemoglobin saturation.

a pulse oximeter at home over the last 6 months? If yes, how did you use it?

\section{Outcome measures}

Outcome measures will be collected at baseline (two visits, 1 week apart), 3 and 6 months following randomisation (table 1), by an assessor who is blinded to group allocation. Three months were selected as this is sufficient to achieve change in the primary outcome ${ }^{26}$ and 6 months will provide robust data for economic analyses.

The primary outcome is change in physical activity, measured by the number of steps per day. Steps per day is an objective measure of physical activity in people with fILD that has strong relationships to respiratory function, exercise capacity, exertional desaturation, HRQL and fatigue. ${ }^{12}$ Physical inactivity, defined as less than 3300 steps per day, is associated with poor survival $(30 \%$ vs $70 \%$ over 3 years). ${ }^{5}$ Steps per day is responsive to changes following non-pharmacological interventions in chronic lung disease ${ }^{26}$ and the minimal important difference has been defined as 599 steps. ${ }^{27}$ Steps per day is a direct measure of how a patient functions in daily life and thus fulfils the criteria for meaningful endpoints in fILD clinical trials. ${ }^{28}$ Steps per day will be measured using the StepWatch activity monitor (SAM) (Modus Health, Washington DC, USA) which is reliable and valid in chronic lung disease. ${ }^{29}$ It accurately detects slow walking speeds and is sensitive to small changes in step rate. ${ }^{30}$ The SAM will be worn on the ankle continuously for 7 days (except for bathing) between the first and second baseline visits, and then for 7 days following the 3-month and 6-month assessments. Seven days of monitoring is required for optimum reliability. $^{29}$

Secondary outcomes are change in functional exercise capacity, HRQL, breathlessness, fatigue, anxiety, depression, time spent in moderate to vigorous physical activity, sedentary time, oxygen saturation in daily life, and plasma markers of skeletal muscle metabolism, systemic inflammation and oxidative stress.

Functional exercise capacity will be measured with the 6-minute walk distance ${ }^{31}$ which is responsive to change with acute administration of oxygen ${ }^{13}$ and is a strong predictor of survival in fILD. ${ }^{32}$ The $6 \mathrm{MWT}$ will be performed according to international standards, including performance of two tests at each time point to control for the known learning effect, with the best distance recorded. ${ }^{31}$ All tests will be performed breathing room air so that a valid comparison can be made across time points, ${ }^{31}$ and to maintain blinding. HRQL will be measured using three instruments: the St George's Respiratory Questionnaire, a disease specific HRQL measure that is valid and responsive in $\mathrm{fILD}^{33}$; the EQ-5D-5L, a validated generic quality of life measure which is used to derive health utilities for economic analyses; and the King's Brief Interstitial Lung Disease Questionnaire, a validated disease-specific health status questionnaire. ${ }^{14}$ The Dyspnoea-12 will be used to capture both the physical and affective components of breathlessness and is a reliable and valid questionnaire in fILD. ${ }^{34}$ Fatigue will be measured with the Fatigue Severity Scale, a valid and sensitive questionnaire in fILD. ${ }^{35}$ Anxiety and depression will be evaluated using the Hospital Anxiety and Depression Scale, a validated 
and widely used tool for assessing psychological distress. Anxiety and depression are common in fILD and are increased in users of LTOT. ${ }^{36}$ The GeneActiv (GENEActiv, Cambridgeshire, UK) will measure time spent in moderate to vigorous physical activity and sedentary time. This wrist-worn, triaxial accelerometer has been validated in IPF. ${ }^{37}$ Seven days of monitoring are required to accurately capture all activity intensities. ${ }^{38}$ Oxygen saturation in daily life will be measured using a Nonin 3150 Wrist Oximeter. The wrist oximeter will be worn during waking hours on 2 consecutive weekdays, with the display turned off so that participants remain blinded to their oxygen saturation during POC use. Examination of plasma markers of skeletal muscle metabolism (xanthine, hypoxanthine); systemic inflammation (interleukin-6, tumour necrosis factor-a, $\mathrm{C}$ reactive protein) and oxidative stress (8-isoprostane, thiobarbiuric acid reactive substrates), as previously published, ${ }^{11}$ will be performed.

\section{Health economic analyses}

We will undertake a comparison of per-person costs, including direct (health system) and indirect (personal) healthcare costs, of ambulatory oxygen therapy compared with air. Direct costs will include staff time, consumables, communications and overheads. Intervention costs will include staff inputs by duration, type and resource use (including troubleshooting and support) and equipment (POC and consumables). Personal costs will include transportation, travel time and impact of the intervention on the economic activities of other household members. Health system costs will include visits to the general practitioner, specialist or emergency department, including any telemedicine visits; use of chronic disease services and hospitalisation. We will collect healthcare utilisation data from hospital records, Medicare Benefits Schedule and Pharmaceutical Benefits Scheme data (Australia) and the National Patient Registry of the Swedish Board of Health and Welfare, as well as directly from participants via monthly telephone calls. Sensitivity analyses will use different assumptions about personal healthcare costs across countries.

An incremental cost-effectiveness analysis will be undertaken to compare differences in costs with differences in: (a) quality-adjusted life years (QALYs): a single preferencebased utility score will be derived from the EQ-5D-5L. This will be converted to QALYs on the assumption that the duration of each status is exactly one-half of the time between two measurement intervals ${ }^{39}$; (b) the number of hospital admissions per enrolled person in the 6-month follow-up period. The indicator will be the incremental cost of averting an additional hospitalisation.

\section{Schedule of assessments}

Outcomes will be obtained by a blinded assessor at baseline, 3 months and 6 months (table 1). At the baseline visit, an arterial blood gas will be performed in participants with resting $\mathrm{SpO}_{2}<93 \%$ to exclude resting hypoxaemia, as this is an indication for LTOT. At each assessment two 6MWTs will be performed according to international standards with continuous pulse oximetry ${ }^{31}$ while the participant breathes room air. The nadir oxyhaemoglobin from the longest 6MWT will be used to determine eligibility at baseline. ${ }^{31}$ Questionnaires will be administered and blood for biomarkers will be obtained. The StepWatch and the GENEActiv activity monitors will be given to participants to wear over the following 7 days and the Nonin 3150 Wrist Oximeter will be worn on 2 consecutive weekdays. The monitors will be returned to the investigators by post. Spirometry is performed every 6 months in usual care, to document disease progression. To minimise patient burden, we will not repeat this test separately for the trial.

\section{Safety and adverse events}

Adverse events will be defined according to Good Clinical Practice guidelines. Adverse events of specific interest will be defined according to the criteria used in the recent Long-term Oxygen Treatment Trial: worsening of fILD (worsening of lung function, development of resting hypoxaemia); exacerbation of fILD; burns (from smoking while using a POC, using the POC around an open flame or equipment that sparks); nosebleed or dry nose; musculoskeletal injury from tripping on a POC; hospitalisation or death. ${ }^{40}$ Adverse events will be identified during monthly telephone calls and three monthly assessment visits, or by reports from the treating medical team. Participants who experience an adverse event will receive all necessary medical care from their local healthcare team.

\section{Sample size}

A total of 220 participants (110 per group) will provide $80 \%$ power to detect, at the two-sided $5 \%$ level, a clinically important difference between groups in the primary outcome of 599 steps per day. ${ }^{27}$ This assumes an SD of 1582 steps, based on physical activity data previously collected at our centre in 52 patients with fILD. Our previous trials had less than $10 \%$ attrition. ${ }^{1541}$ Previous experience suggests that $5 \%$ of participants could start LTOT (and cease POC use) over 6 months. We will therefore randomise 260 participants to ensure that 220 participants complete the study.

Over 1000 patients with fILD are currently managed at our centres, with an additional 300 new patients seen each year. Approximately half of these patients exhibit exertional desaturation and are not using LTOT. $^{42}$ Based on the rate of recruitment in our feasibility study, ${ }^{21}$ we anticipate recruiting the required sample of 260 participants over 3 years.

\section{Analysis}

Mean differences for continuous variables will be analysed using linear mixed models, controlling for baseline values as required. Generalised linear mixed models will be used for binary or count outcomes. Estimates will be presented with $95 \%$ CIs and two-sided $\mathrm{p}$ values reported. Results 
will be displayed graphically where it will illuminate. All data will be analysed by intention to treat, including all randomised participants in the groups to which they were allocated, regardless of adherence.

\section{Data integrity and management}

Hardcopy data collection forms will be stored in a locked filing cabinet within a locked office, and electronic data will be stored in a purpose-built online database (www. adeptrs.com), with encryption and password protection. The online database will be protected by encryption enabled at up to 256-bits and SSL (Secure Sockets Layer) certificate, and hosted on a dedicated SSL cluster. No identifying information will be stored in the online database or on hardcopy data forms. Electronic data for all sites will be accessible by the principal investigator and the trial coordinator. Site-specific investigators will only have access to data relating to their individual site. Information will be stored indefinitely, in accordance with Human Research Ethics Committees requirements for interventional studies.

\section{Data monitoring}

The Data Safety and Monitoring Committee (DSMB) will meet two times yearly, chaired by a respiratory physician who is independent of the study team and trial sites. The DSMB will include a biostatistician. The DSMB will report its findings to the trial steering committee, consisting of the chief investigators.

\section{Ethics and dissemination}

Ethical approval has been granted in Australia by Alfred Hospital Human Research Ethics Committee (HREC/18/Alfred/42) with governance approval at all Australian sites, and in Sweden (Lund Dnr: 2019-02963). The study will be conducted and reported according to the SPIRIT guidelines ${ }^{22}$ and the Consolidated Standards of Reporting Trials statement. ${ }^{43}$ Results will be published in peer-reviewed journals and presented at conferences. We will also disseminate our results to people with fILD through lay publications and seminars.

\section{DISCUSSION}

This study will recruit people with fILD who desaturate during walking, a group that represents half of all patients with fILD and over $85 \%$ of those with severe disease. ${ }^{42}$ People with fILD experience distressing breathlessness, cough and fatigue; loss of independence and life roles; financial strain and unpleasant treatment side effects. ${ }^{44}$ Many have few treatment options. This multicentre trial will examine the benefits and costs of ambulatory oxygen, delivered using a POC, in people with fILD and exertional desaturation. Recruitment across six sites and two countries will enhance external validity. Use of a sham POC allows effective blinding, a feature frequently missing from trials of oxygen therapy, thus substantially reducing the risk of bias. The primary outcome is steps per day, a direct measure of patient function in daily life. The study also includes a comprehensive economic analysis, to inform future funding and policy decisions.

Limitations of our trial include a follow-up period of 6 months, so longer term outcomes of ambulatory oxygen will not be evaluated. A diagnosis of fILD by a multidisciplinary team (MDT) is not required for inclusion, which may reduce certainty regarding the fILD subtypes included, however it is common practice in our centres that diagnosis is made by an MDT. Patients who have previously used ambulatory oxygen therapy are not excluded, which could affect participant expectations and response to treatment with the POC; however we have previously shown that less than $30 \%$ of eligible patients with fILD are currently using ambulatory oxygen, ${ }^{42}$ so we anticipate that the majority will be naïve to this treatment.

Recent years have brought a new sense of hope for people with fILD, with the advent of antifibrotic therapies that slow the progression of disease and have revolutionised the approach to treatment. ${ }^{46}{ }^{47}$ However, this hope has been tempered by their lack of impact on patient-centred outcomes, including HRQL. Interventions that improve how people with fILD feel and function are urgently needed. Ambulatory oxygen is currently available to some patients with fILD, but patient access is inconsistent across health systems, reflecting the lack of evidence underpinning this treatment. For patients with fILD, ambulatory oxygen has potential benefits but also potential burdens. ${ }^{19}$ This clinical trial will provide muchneeded evidence to underpin decisions by health professionals and patients regarding prescription and ongoing use of ambulatory oxygen. If successful, the findings of this trial can be rapidly incorporated into clinical guidelines and implemented into clinical practice across the world.

\section{Trial status}

Recruitment commenced in July 2019.

\section{Author affiliations}

${ }^{1}$ Department of Physiotherapy, Alfred Health, Melbourne, Victoria, Australia ${ }^{2}$ Department of Allergy, Immunology and Respiratory Medicine, Monash University, Melbourne, Victoria, Australia

${ }^{3}$ Institute for Breathing and Sleep, Melbourne, Victoria, Australia

${ }^{4}$ NHMRC Centre of Research Excellence in Pulmonary Fibrosis, Camperdown, New South Wales, Australia

${ }^{5}$ Department of Respiratory Medicine, Royal Prince Alfred Hospital, Sydney, New South Wales, Australia

${ }^{6}$ Central Clinical School, The University of Sydney, Sydney, New South Wales, Australia

${ }^{7}$ School of Clinical Medicine, The University of Queensland, Brisbane, Queensland, Australia

${ }^{8}$ Queensland Lung Transplant Service, The Prince Charles Hospital, Brisbane, Queensland, Australia

${ }^{9}$ Health Economics Research Group, Menzies Institute for Medical Research, The University of Tasmania, Hobart, Tasmania, Australia

${ }^{10}$ Centre for Health Policy, School of Population and Global Health, The University of Melbourne, Melbourne, Victoria, Australia

${ }^{11}$ Respiratory Medicine and Allergology, Department of Clinical Sciences, Faculty of Medicine, Lund University, Lund, Sweden

${ }^{12}$ Department of Respiratory and Sleep Medicine, Alfred Health, Melbourne, Victoria, Australia 
${ }^{13}$ Faculty of Medicine, University of Melbourne, Melbourne, Victoria, Australia

${ }^{14}$ Department of Respiratory and Sleep Medicine, Austin Health, Melbourne, Victoria, Australia

${ }^{15}$ Statistical Consulting Centre, University of Melbourne, Melbourne, Victoria, Australia

${ }^{16}$ School of Health and Biomedical Sciences, RMIT University, Bundoora, Melbourne, Australia

${ }^{17}$ Respiratory Medicine Unit, Department of Medicine Solna and Center for

Molecular Medicine, Karolinska Institutet, Stockholm, Sweden

${ }^{18}$ Department of Respiratory Medicine and Allergy, Karolinska University Hospital, Stockholm, Sweden

${ }^{19}$ Department of Physiotherapy, Austin Health, Melbourne, Victoria, Australia

${ }^{20}$ Department of Respiratory Medicine, Royal Melbourne Hospital, Melbourne,

Victoria, Australia

${ }^{21}$ Physiotherapy Department, The Prince Charles Hospital, Brisbane, Queensland, Australia

Contributors AEH led the study design and funding application and, as chief investigator, has oversight for the trial. AEH and CFM conceived the original idea for the study. AEH and MH wrote the initial draft of the study protocol. TC, DCC, AJP, MPE, IG, NSLG, GH, MS, LB and RV contributed to protocol development and refined the trial design. GH and AEH planned the statistical analyses. YHK, RV, LD, LT, JP and JW collected pilot data and contributed to selection of outcomes. All authors conducted the PFOX trial, critically revised the manuscript and approved the final version for publication.

Funding This work was supported by National Health and Medical Research Council (Australia) grant 1139953 and an unrestricted grant from the Swedish Society of Medicine (SLS-786791). The authors acknowledge Linde's Healthcare Centre of Excellence for advice on the selection and sourcing of concentrators used in the study, particularly Syed Jafri, Humberto Gomes and Urmi Richardson.

Competing interests All authors report non-financial support from BOC Australia in the delivery of the trial devices. AEH, YHK, LT, NSLG and CFM report non-financial support from Air Liquide Healthcare, outside the submitted work. YHK reports grants and personal fees from Boehringer Ingelheim, and personal fees from Roche, outside the submitted work. MS received research grants from Boehringer Ingelheim and Roche, outside the submitted work

Patient consent for publication Not required.

Provenance and peer review Not commissioned; externally peer reviewed.

Supplemental material This content has been supplied by the author(s). It has not been vetted by BMJ Publishing Group Limited (BMJ) and may not have been peer-reviewed. Any opinions or recommendations discussed are solely those of the author(s) and are not endorsed by BMJ. BMJ disclaims all liability and responsibility arising from any reliance placed on the content. Where the content includes any translated material, BMJ does not warrant the accuracy and reliability of the translations (including but not limited to local regulations, clinical guidelines, terminology, drug names and drug dosages), and is not responsible for any error and/or omissions arising from translation and adaptation or otherwise.

Open access This is an open access article distributed in accordance with the Creative Commons Attribution Non Commercial (CC BY-NC 4.0) license, which permits others to distribute, remix, adapt, build upon this work non-commercially, and license their derivative works on different terms, provided the original work is properly cited, appropriate credit is given, any changes made indicated, and the use is non-commercial. See: http://creativecommons.org/licenses/by-nc/4.0/.

ORCID iD

Anne E Holland http://orcid.org/0000-0003-2061-845X

\section{REFERENCES}

1 Cottin V, Wollin L, Fischer A et al. Fibrosing interstitial lung diseases: knowns and unknowns. Eur Respir Rev 2019;28. doi:10.1183/16000617.0100-2018. [Epub ahead of print: 31 Mar 2019].

2 Papakosta D, Pitsiou G, Daniil Z, et al. Prevalence of pulmonary hypertension in patients with idiopathic pulmonary fibrosis: correlation with physiological parameters. Lung 2011;189:391-9.

3 Corte TJ, Wort SJ, Wells AU. Pulmonary hypertension in idiopathic pulmonary fibrosis: a review. Sarcoidosis Vasc Diffuse Lung Dis 2009;26:7-19.
4 Lama VN, Flaherty KR, Toews GB, et al. Prognostic value of desaturation during a 6-minute walk test in idiopathic interstitial pneumonia. Am J Respir Crit Care Med 2003;168:1084-90.

5 Wallaert B, Monge E, Le Rouzic O, et al. Physical activity in daily life of patients with fibrotic idiopathic interstitial pneumonia. Chest 2013;144:1652-8.

6 Hardinge M, Annandale J, Bourne S, et al. British thoracic Society guidelines for home oxygen use in adults. Thorax 2015;70 Suppl 1:i1-43.

7 Khor YH, Goh NSL, McDonald CF, et al. Oxygen therapy for interstitial lung disease: physicians' perceptions and experiences. Ann Am Thorac Soc 2017:14:1772-8.

8 Chan L, Giardino N, Rubenfeld G, et al. Geographic differences in use of home oxygen for obstructive lung disease: a national Medicare study. J Rural Health 2010;26:139-45.

9 Lacasse Y, Bernard S, Maltais F. Eligibility for home oxygen programs and funding across Canada. Can Respir J 2015;22:324-30.

10 McDonald CF, Whyte K, Jenkins S, et al. Clinical practice guideline on adult domiciliary oxygen therapy: executive summary from the thoracic Society of Australia and New Zealand. Respirology 2016;21:76-8.

11 Dowman LM, McDonald CF, Bozinovski S, et al. Greater endurance capacity and improved dyspnoea with acute oxygen supplementation in idiopathic pulmonary fibrosis patients without resting hypoxaemia. Respirology 2017:22:957-64.

12 Bahmer T, Kirsten A-M, Waschki B, et al. Clinical correlates of reduced physical activity in idiopathic pulmonary fibrosis. Respiration 2016;91:497-502.

13 Bell EC, Cox NS, Goh N, et al. Oxygen therapy for interstitial lung disease: a systematic review. Eur Respir Rev 2017;26:160080.

14 Visca D, Mori L, Tsipouri V, et al. Effect of ambulatory oxygen on quality of life for patients with fibrotic lung disease (AmbOx): a prospective, open-label, mixed-method, crossover randomised controlled trial. Lancet Respir Med 2018;6:759-70.

15 Moore RP, Berlowitz DJ, Denehy L, et al. A randomised trial of domiciliary, ambulatory oxygen in patients with COPD and dyspnoea but without resting hypoxaemia. Thorax 2011;66:32-7.

16 Khor YH, McDonald CF, Hazard A, Symons K, et al. Portable oxygen concentrators versus oxygen cylinder during walking in interstitial lung disease: a randomized crossover trial. Respirology 2017:22:1598-603.

17 Schoenheit G, Becattelli I, Cohen AH. Living with idiopathic pulmonary fibrosis: an in-depth qualitative survey of European patients. Chron Respir Dis 2011;8:225-31.

18 Duck A, Spencer LG, Bailey S, et al. Perceptions, experiences and needs of patients with idiopathic pulmonary fibrosis. J Adv Nurs 2015;71:1055-65.

19 Khor YH, Goh NSL, McDonald CF, et al. Oxygen therapy for interstitial lung disease. A mismatch between patient expectations and experiences. Ann Am Thorac Soc 2017;14:888-95.

20 Morell F, Esser D, Lim J, et al. Treatment patterns, resource use and costs of idiopathic pulmonary fibrosis in Spain-results of a Delphi Panel. BMC Pulm Med 2016;16:7.

21 Khor YH, Holland AE, Goh NSL, et al. Ambulatory oxygen in fibrotic interstitial lung disease: a pilot, randomized, blinded, shamcontrolled trial. Chest 2020;158:234-44.

22 Chan A-W, Tetzlaff JM, Altman DG, et al. Spirit 2013 statement: defining standard protocol items for clinical trials. Ann Intern Med 2013;158:200-7.

23 Flaherty KR, Brown KK, Wells AU, et al. Design of the PF-ILD trial: a double-blind, randomised, placebo-controlled phase III trial of nintedanib in patients with progressive fibrosing interstitial lung disease. BMJ Open Respir Res 2017;4:e000212.

24 Raghu G, Collard HR, Egan JJ, et al. An official ATS/ERS/JRS/ALAT statement: idiopathic pulmonary fibrosis: evidence-based guidelines for diagnosis and management. Am J Respir Crit Care Med 2011:183:788-824.

25 Nishiyama $\mathrm{O}$, Taniguchi $\mathrm{H}$, Kondoh $\mathrm{Y}$, et al. Health-related quality of life in patients with idiopathic pulmonary fibrosis. What is the main contributing factor? Respir Med 2005:99:408-14.

26 Lahham A, McDonald CF, Holland AE. Exercise training alone or with the addition of activity counseling improves physical activity levels in COPD: a systematic review and meta-analysis of randomized controlled trials. Int J Chron Obstruct Pulmon Dis 2016;11:3121-36

27 Demeyer H, Burtin C, Hornikx M, et al. The minimal important difference in physical activity in patients with COPD. PLoS One 2016;11:e0154587.

28 Raghu G, Collard HR, Anstrom KJ, et al. Idiopathic pulmonary fibrosis: clinically meaningful primary endpoints in phase 3 clinical trials. Am J Respir Crit Care Med 2012;185:1044-8. 
29 Danilack VA, Okunbor O, Richardson CR, et al. Performance of a pedometer to measure physical activity in a U.S. cohort with chronic obstructive pulmonary disease. J Rehabil Res Dev 2015;52:333-42.

30 Cindy Ng LW, Jenkins S, Hill K. Accuracy and responsiveness of the stepwatch activity monitor and activPAL in patients with COPD when walking with and without a rollator. Disabil Rehabil 2012;34:1317-22.

31 Holland AE, Spruit MA, Troosters T, et al. An official European respiratory Society/American thoracic Society technical standard: field walking tests in chronic respiratory disease. Eur Respir $J$ 2014:44:1428-46.

32 Flaherty KR, Andrei A-C, Murray S, et al. Idiopathic pulmonary fibrosis: prognostic value of changes in physiology and six-minutewalk test. Am J Respir Crit Care Med 2006;174:803-9.

33 Swigris JJ, Brown KK, Behr J, et al. The SF-36 and SGRQ: validity and first look at minimum important differences in IPF. Respir Med 2010;104:296-304.

34 Yorke J, Swigris J, Russell A-M, et al. Dyspnea-12 is a valid and reliable measure of breathlessness in patients with interstitial lung disease. Chest 2011;139:159-64.

35 Swigris JJ, Fairclough DL, Morrison M, et al. Benefits of pulmonary rehabilitation in idiopathic pulmonary fibrosis. Respir Care 2011;56:783-9.

36 Holland AE, Fiore JF, Bell EC, et al. Dyspnoea and comorbidity contribute to anxiety and depression in interstitial lung disease. Respirology 2014;19:1215-21.

37 Atkins $\mathrm{C}$, Baxter $\mathrm{M}$, Jones $\mathrm{A}$, et al. Measuring sedentary behaviors in patients with idiopathic pulmonary fibrosis using wrist-worn accelerometers. Clin Respir J 2018;12:746-53.

38 Dillon CB, Fitzgerald AP, Kearney PM, et al. Number of days required to estimate habitual activity using wrist-worn
GENEactiv accelerometer: a cross-sectional study. PLoS One 2016;11:e0109913.

39 Sinnott PL, Joyce VR, Barnett PG. Preference measurement in economic analysis. Guidebook. Menlo Park CA, VA Palo Alto: Health Economics Resource Center, 2007.

40 Long-Term Oxygen Treatment Trial Research Group, Albert RK, Au $\mathrm{DH}$, et al. A randomized trial of long-term oxygen for COPD with moderate desaturation. N Engl J Med 2016;375:1617-27.

41 Holland AE, Mahal A, Hill CJ, et al. Home-based rehabilitation for COPD using minimal resources: a randomised, controlled equivalence trial. Thorax 2017;72:57-65.

42 Khor YH, Goh NS, Glaspole I, et al. Exertional desaturation and prescription of ambulatory oxygen therapy in interstitial lung disease. Respir Care 2019;64:299-306.

43 Schulz KF, Altman DG, Moher D, et al. Consort 2010 statement: updated guidelines for reporting parallel group randomized trials. Ann Intern Med 2010;152:726-32.

44 Swigris JJ, Stewart AL, Gould MK, et al. Patients' perspectives on how idiopathic pulmonary fibrosis affects the quality of their lives. Health Qual Life Outcomes 2005;3:61.

45 Holland AE, Fiore JF, Goh N, et al. Be honest and help me prepare for the future: what people with interstitial lung disease want from education in pulmonary rehabilitation. Chron Respir Dis 2015;12:93-101.

46 Richeldi L, du Bois RM, Raghu G, et al. Efficacy and safety of nintedanib in idiopathic pulmonary fibrosis. N Engl $\mathrm{J} \mathrm{Med}$ 2014;370:2071-82.

47 King TE, Bradford WZ, Castro-Bernardini S, et al. A phase 3 trial of pirfenidone in patients with idiopathic pulmonary fibrosis. $N \mathrm{Engl} J$ Med 2014;370:2083-92. 